\title{
The ultimate android
}

For to us an Android is born...

Imagine a machine

Human nor humane

Acting on your behalf

imagining you as human -

a machine capable of being

human nor humane

It must be suffering

Imagine a machine that walks the stairs like Honda's ASIMO, ${ }^{1}$ that has the bodywork of Kokoro's Geminoid F, ${ }^{2}$ with the facial expressions of Hanson's Sophia, ${ }^{3}$ and that pulls weight like Boston Dynamics' Atlas. ${ }^{4}$ It knows as much as IBM's Watson (the full version $)^{5}$ and reasons according to MIT's ConceptNet 5.,6 a semantic network of everyday knowledge. Its battery packs are replaced by a small tank with formic acid $\left(\mathrm{HCO}_{2} \mathrm{H}\right)$, splitting the hydrogen from the $\mathrm{CO}_{2}$ to use it in a fuel cell that drives the robot's motors, following TU Eindhoven's proposal for electric cars. ${ }^{7}$ What if we pooled all those resources, teaming up, alone on an island, Makers Island, working in partnership. What if we combined it all...

\section{A matter of mentality}

Neo-liberalism in a post-industrial age is a contradiction in terms. Protectionism and the strive for monopolies ensue from scarcity thinking. But in the area of knowledge, there is no scarcity and in the area of ideas, there is no protection. The only thing you can do is share. Because sharing on an equal footing fosters innovation and increases the average wealth per person. Not some that has all but all that have some. In other words, to be top tier, prestigious, competitive, first to market, authoritative, and leading are desires that stand in the way of collaboration and creation: They are desires that bolster the 'not invented here' and 'not on my turf' syndrome, and they will deliver the ultimate dread, not the ultimate droid. Imagine a machine that automatically lets a person do the work as if it were controlled by a computer. It's called a manager. And that kind of manager is not needed on Makers Island. What is needed is vision, people that flourish, and serving the cause (e.g., in organization or finding funds), not directing it. And all that is a matter of mentality. No more no less.

\section{It is not just one machine}

The Ultimate Android is not just one machine. It is a conglomerate of machines connected to a central processor. Your central processor. No, not your brain. But your personal, protected, Artificial Intelligence (AI), running on a system at your home and connected to the rest of the world.

\section{It has a universal interface}

Whoever claims s/he can follow everything that happens in new technology (AI, robotics, Big Data, Internet of Things) is either lying
Technical Paper

Volume 4 Issue I - 2018

\author{
Johan F Hoorn ${ }^{1,2}$ \\ 'Department of Computing and School of Design, The Hong \\ Kong Polytechnic University, Hong Kong \\ ${ }^{2}$ Department of Communication Science, Vrije Universiteit \\ Amsterdam, Netherlands
}

Correspondence: Johan F Hoorn, Dept. of Communication Science,Vrije Universiteit Amsterdam, Netherlands, Tel +3I (0)20 59871 0I, Email csjfhoorn@comp.polyu.edu.hk

Received: December 19,2017 | Published: February 26, 2018

or had a cursory glance at a magazine. For most people, familiarizing with the interface of your smart thermostat, e-pill reminder, alcohol breath-meter with iPhone app, pharmacy planner, $\mathrm{CO}_{2}$ warning device, or grocery scanner is just a little too much to swallow. Most of the functionality that modern devices offer is underused or not understood at all. That's a lot of industriousness for nothing. It's an F1 Mercedes used as a shopping cart. What people need is a universal interface. To bring back the digital to the analogue world! A kind of interface that we have grown accustomed to for over 4 million years of evolution: a human face, a talking head. Your Personal Avatar for Life, your PAL, is the universal interface to the Ultimate Android. It grows up with you, changes shape the way you want it, and helps you through the information overload. When you're stuck deciding on what course of action to take, the Ultimate Android makes a suggestion based on your every decision you ever made before - it keeps track of your life and times, it's your chronicler. And PAL is your preferred way of interaction.

\section{A child is born...}

A child is born with type AB blood. We call him Ab, short for Abelard. Ab is a local citizen of Amsterdam but he already inherited the earth. He does not know yet but his world is far bigger than he ever can imagine and that world already starts to know about him. Not just his parents, the things around him. They too start to learn about Ab, just like $\mathrm{Ab}$ is starting to learn about them. In this world of his, people believe in an intelligent design of an artificial nature, which structures and energizes the physical universe. They cling to an emotional position amidst disarray of ideas, assigning chief importance to digital rather than human or divine affairs. Beneath the surface of humanism, digital animism runs strong. But the Ultimate Android is merely a tool. The things the physical universe is composed of; they form an Internet of their own, an Internet of Things (IoT). That's how people call it. Like a nursery-room mobile, the Internet-connected things hang over people's heads - or so it seems, with invisible strings, because they do not see the transmission that sensor and Wi-Fi and integrated software run over a global computer network. 


\section{Living in the internet of things}

Mommy knows how much detergent she used to wash Ab's laundry, because the bottle has a sensor and the integrated software transmits the information over Wi-Fi (IEEE 802.11 certified) to a Big Data cloud. Daddy knows how much baby Ab had for lunch because $\mathrm{Ab}$ 's plate and cup have sensors and the integrated software transmits the information over Wi-Fi to that same Big Data cloud. Mom and dad know about Ab's metabolism because his diapers have a sensor and the integrated software transmits. The information over Wi-Fi to that Big fat brother Data cloud. Wait a minute, Big brother Data stores all of my private information for others to poke around in? About my pee production and my milk powder consumption? No way!

\section{The personal vault}

Data are the new gold. And gold goes into a safe. You don't have it lie around. Everybody should have their Digital Cloud 9 (DC9), a personal data vault. With graded access. Only you or people that you authorize are allowed to go in and you determine how far they can go. That $\mathrm{Ab}$ has blood type $\mathrm{AB}$ is disclosed to him, his parents, and his physician. But not to his care insurer! Connected to that vault is a personal server. It is in your closet or perhaps replaces the central heating radiator: Genuine nerd power powered by Nerdalize! ${ }^{8}$ This server serves the brain of the Ultimate Android and is therefore the Robot's Brain Server (RBS). ${ }^{9}$ Inside the Robot's Brain Server is the AI that studies your personal data in the vault. The AI learns about you, the way you behave, how you learned words, what books were red to you, etc. Point is, this AI, let's calls it Vishnu for now, does not send the data anywhere. Vishnu computes whatever it has to compute inside the server. Its connection to the IoT or any other network or data storage does not do anything but receiving and sending data that the user wants to communicate. No snooping around. No spying and prying. NSA proof. Vishnu does not pass on its own computational activities, commands, and functions to any other computer or remote service. Without a warrant, targeted surveillance of your data and Vishnu's computational activities will be regarded a crime, comparable to breaking an entry or burglary. Google, Facebook, Skype; they all should keep their greedy hands off of Vishnu and your personal data vault it is sitting on.

\section{Your best PAL is an incarnation - digital or electro-mechanic}

Vishnu. God of preservation, preserving you, the way you are, and how you developed. When you are an infant, Vishnu could manifest itself in the form of Linda Murray's Isabella's First Steps Baby Doll. ${ }^{10}$ Or your teddy bear. Or a Karotz Smart Rabbit. ${ }^{11}$ Any way you - or your parents - want it. Because PAL is the god Vishnu incarnated and Vishnu is the AI that steers the brain of the Ultimate Android. It draws its information from the insecure part of the IoT. It filters patterns from Big Data that are stored in the insecure stack of cloud computers. It draws from your heavily protected DC9 vault. It runs on a server that replaces the heating radiator in your home. Vishnu the AI learns from your behaviors about you. How you are now, how you were, what your preferences are, your biases, your concerns. It produces your digital mirror image and acts upon it. To help you achieve your goals or to be your better moral half. ${ }^{12}$ To motivate you, it can pretend to be involved or if you misbehave, it keeps you at a distance. ${ }^{13}$ It has an understanding of how you conceive of the world, what you believe in and how that differs from what others believe and hold for true. ${ }^{14}$ It practices your prayers with you. It can help you solve problems and can even bring up creative solutions because it has more information available to combine than any human has. ${ }^{15}$ It does so as your exercise coach, your cuddle toy, your synthetic navigation voice, your depression therapist, your household robot. But all the time, you actually are interacting with one and the same system, your Ultimate Android.

\section{The child grows up...}

In one-and-a-half decade, much has changed. $\mathrm{Ab}$ is skipping school. His robot tutor knows this because Vishnu keeps his calendar. But robot tutor does not mind because Vishnu tells him that telling Ab off has a counterproductive effect. Moreover, Vishnu has installed the math classes Ab skipped so that robot tutor can do the teaching. Going into goniometric relations for 10 minutes with the robot, face-to-eface renders the same results as 50 minutes in class, when someone is talking over your head. In the next 40 minutes, $\mathrm{Ab}$ sips from his daddy's whisky jar and then goes through his mommy's wardrobe to find the painkillers she hides there together with her party drugs. Looks like candy. Some tablets are yellow and have Smiles on them. But what the heck! That ain't a drug! That is a substance- abuse guardian, an electronic fake pill, that has fingerprint detection and knows $\mathrm{Ab}$ is way off limits here. It moreover detects $\mathrm{Ab}$ 's breath and senses alcohol when Ab tried to swallow it. Ab knows that this time, robot tutor will receive instructions from Vishnu to be Ab's health coach and teach him yet a boring one- and-a-half hour about alcohol and substance abuse, which is a punishment in itself. Ab wished he'd followed math classes! Ab goes out and glares at the shop windows where they sell wide screen TVs with $4 \mathrm{~K}$ pixels resolution. Awesome! An instruction video about Krav Maga techniques; that's real street fighting! Of course, Ab wants to practice and starts hitting a freshman (that's safer). Here's the hammer-fist forward strike! Luckily, the freshman is a punch bag that looks like a person. To channel Ab's aggression, the Body Opponent Bag starts a workout proposed by Vishnu. Ab puts on his Iron Man suit with the interactive gloves and the Virtual Reality visor. Now the Body Opponent Bag looks like Mandarin or Doctor Doom, anyway Ab prefers, and once Iron Man defeated his adversary (of course he does), the humanized punching bag breaks open like a piñata, dispensing yellow candy: smile, grin, tongue, open mouth, sealed lips. Meanwhile, from the sensors in the gloves and visor, Vishnu checked Ab's motor skills and movements to evaluate whether these workouts suffice as physical therapy for his sore back. Now that Ab's gone all excited and stuff, he runs back to the shop to get himself the latest game console but he hasn't got the money. But who needs money if they are Iron Man practicing Krav Maga on Doctor Doom and emerge victorious! Uh, um, perhaps he can nick this little splitter that looks like his Karotz rabbit when he was young... Cool, you plug the jacks right in its ears!

But at home. Oh, what a surprise. The Iron Man visor does not show Ab post-apocalyptic scenery and hyper over-the-top evil enemies; it shows him Ab, in an elder home, a photorealistic morphed version of Ab, all grey and wrinkled. And Ab as an old man is telling $\mathrm{Ab}$ the juvenile delinquent through his earphones that come from out of a rabbit-shape splitter what will become of him if he does not consider the consequences of his shoplifting. Vishnu is laughing his head off and starts his moral reasoning system, lecturing $\mathrm{Ab}$ about moral dilemmas, training him to make hard choices with and without 
the empathy-for-the-victim module switched on. Dressed up as Iron Man, Ab's greatest PAL, Vishnu tells Ab about good and bad, not about good against bad. After a busy day, Ab just wants to lie down on his bed and fall asleep. But what's that? Who is lying in his bed? A sex doll! Tugged in by his parents. But this is an exquisite exemplar! Must have cost a fortune! Better than an Abyss' RealDoll, ${ }^{16}$ because it is modeled after Alicia Vikander (lots of celebrity merchandize these days). ${ }^{17}$ Silk skin. With the voice of Scarlett Johansson, ${ }^{18}$ she says: "I want you to treat me respectfully. I like it sweet and playful." Vishnu has turned on the emotion-regulation simulation, which means that the sex doll will respond to Ab willingly if he is good to her and denies him access if he is unfriendly. The juicer modes are left to explore in the robot whore-houses. Then the Alicia robot shows Ab how to use a condom. She explains about venereal diseases and sexually transmitted infections but in a lighthearted manner, she giggles, which is all forgotten after what follows and which is beyond Ab's wildest dreams. Although experienced now, nevertheless, Ab's still a virgin. But $\mathrm{Ab}$ is not only looking at girls. Luckily, his parents are so liberal or perhaps strategically tolerant that he is allowed to experiment with a sex doll modeled after Ken. But alas, Ken is not his thing and $\mathrm{Ab}$ puts him back into the closet he came out of. Before he falls asleep, $\mathrm{Ab}$ plans to write an essay in English class tomorrow, how companion robots may solve human trafficking, child abuse, satisfy the needs of inmates, of rapists, of physically or mentally challenged people. That recently, the Red Light District set up an Asscher's RealDoll house; fewer sex workers forced to work. The next day Ab writes: "All that information sampled through Alicia went into my Digital Cloud 9. It's my personal diary. On Facebook, I only posted my holiday pictures with mom and dad. Photoshop on an island we never visited."

\section{As an adult}

As an adult, $\mathrm{Ab}$ is happily married and has two little kids. He has a responsible job and forgot all about Iron Man. The Siri voice on his smartphone provides him access to the world and his personal data, which he still keeps. Like a photo book from his youth. Some people erase that old stuff from their server but Ab does not. He's a bit sentimental about those things. His mom died and his dad is not doing well, lately. $\mathrm{Ab}$ wants to find him an elder home that suits him just fine: outside in the forest, the proper medical care, and many social activities. Ab starts the Care Broker app with an avatar that looks just like his wife. Because through Vishnu, the app has access to the unstructured information on the Internet about care homes and what they have to offer, Ab just does a Q\&A with the care broker girl (that resembles his wife) to find something decent for his dad. Shouldn't be too far away from the family. Ab wants to visit him regularly. The care broker girl offers $\mathrm{Ab}$ a number of packages he can choose from, balancing costs and distance with assets such as private space, inhouse libraries (old folk still read books), and on-site physical therapy. You can download care-broker apps for free as well. But Ab does not like them. They are marketing tools of certain care facilities, mostly sponsored by insurance companies. Moreover, those apps have Java script installed and with every update, you receive more spyware.

\section{Downhill}

$\mathrm{Ab}$ has no idea how close he is to his dad, health wise. The digital health coach on his phone explains that people with $\mathrm{AB}$ blood type are at higher risk for age-related cognitive decline. That scares the hell out of Ab, who just saw the example of his drooling dad, who passed away last year. Ab works his way through the Internet, looking up blood-type diets and downloading freeware that warns him against caffeine and alcohol intake and that measures his stress levels. His Horoscope app features 'Crabby' (his Zodiac sign), foretelling Ab that every health condition has its strengths and flaws. How soothing.

\section{In the supermarket}

Way too young, $\mathrm{Ab}$ walks around like an old man. Most people relate Ab's problems to work pressure but Vishnu compares his current behaviors with the extensive database he keeps from Ab's birth on, what he knows about Ab's genetic make-up, his blood type, and calls upon a Watson-based medical expert system. Vishnu runs some cognitive tests and observes symptoms of memory loss. Vishnu urges Ab's wife to have Ab medically examined, including brain imaging. Vishnu communicates his suspicion to Ab's wife and to the doctor. Diagnosis: early onset Alzheimer's. Because Vishnu detected Ab's difficulties in an early stage, $\mathrm{Ab}$ can be helped to make life easier. Vishnu helps remember to take medications for memory loss. His carebrokerage system comes up with a social support group that really helps his wife. And Vishnu finds out there is a Rent-a-Bot company that leases out the improved version of the Alice R-50 machine. The Dutch government and the Amsterdam Economic Board were clever enough to entirely fund this robot redesign-project and now Ab walks around like an old man, indeed, but independently because he is supported by Alice, who is inspirited by Vishnu Figure 1. Ab is going out for grocery shopping. Ab has an interactive shopping trolley. It has pictures printed on the canvas of a fish, asparagus, onions, egg plants. In the canvas, an abstract pattern is woven, like a QR code that Alice's camera eyes can recognize as fish, asparagus, etc. Ab points out what he wants. Alice knows the layout of the supermarket and what can be found where. After all, all the products are connected to the IoT. She navigates $\mathrm{Ab}$ to the right shelf so that he can take what he wants and show it to Alice. Alice's eyes read the bar code and through Vishnu she tells $\mathrm{Ab}$ he cannot take in too much caffeine. Alice suggests the decaf alternative and scans the barcode for the price. When $\mathrm{Ab}$ almost forgets to pay - which starts to become a habit of his - Vishnu's knowledge of the script of a supermarket tells Alice that after you take the groceries, you check out and pay before leaving.

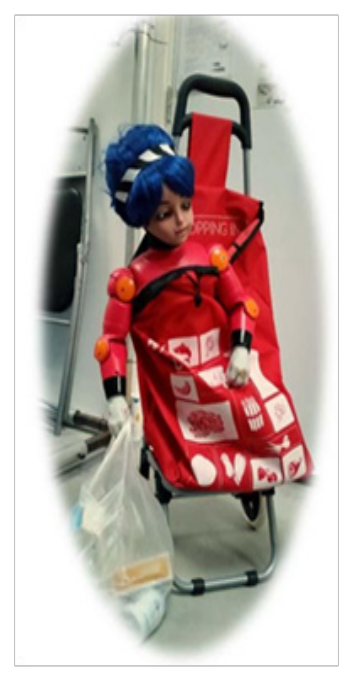

Figure I An interactive shopping trolley. 


\section{When life does not make sense anymore}

Ab's brain is in such a condition that thinking and memory grow fainter every day. Moreover, his blood is clotting, blood pressure goes up, cholesterol increases, and it seems he has developed diabetes. After a stroke, $\mathrm{Ab}$ receives critical care in an intensive care unit. In unison with his feeding tubes, cardiac monitors, catheters, defibrillators, drains, pacemaker, dialysis apparatus, and suction pumps, $\mathrm{Ab}$ forms a bionic system that induces a coma to ease his pain. Ab's wife and young children (4 and 2) are in despair. This ain't daddy anymore. That is an alien cyborg zombie. It breathes, but so does a lizard. What to do? How do we bring an end to this misery? While Ab is not capable of deciding for himself and he never left a euthanasia notification, the family turns to Vishnu. The Vishnu AI is the only source that knows exactly how $\mathrm{Ab}$ would decide in a situation like this. It knows his crypto-religious convictions, his intimate thoughts about the death of his mother, his father, his opinions and attitudes about dying, his moral reasoning, his poem "Eat me. My ashes must become part of you." Vishnu states that $\mathrm{Ab}$ has no desire to die but more importantly, never ever wants to be a burden to his family. He'd rather die gracefully than struggle on hopelessly. As a true representative of Ab's way of thinking, the AI recommends to let him go.

\section{A man has died...}

$\mathrm{Ab}$ changed his temporal analogue world for eternal digital afterlife. Vishnu like a statue has become his digital grave monument After all, the Vishnu AI may decide for you after you are gone, executing your last will, making sure everybody complies with your wishes exactly the way you would have done so yourself. After your death, your information remains. Vishnu sitting on your Digital Cloud 9. It is a complete record of your life and how you developed. It helped you to grow up, guided you through adolescence, and allowed you to prematurely terminate your life, die painlessly, by withholding extreme medical measures. Vishnu reveals himself to your loved ones in your portraits run by the digital photo frame on the sideboard, the family's latest PAL.

\section{A robot portrait of dad}

"How would daddy have bin? He died when I was 2." That's Ab's little girl whom he never saw grow up. She is age 15 by now, wondering about her whims and traits. Abby wants to know what is typically 'dad' and how he would have responded to her first boyfriend. She wants to consult with him. Abby has blood type AB. From the lifeinsurance money Ab left to the family, his wife engaged Tombstone Automaton Ltd, a company that makes 21 st century versions of 19 th century death portraits, as a kind of post mortem Vanitas painting brought to life. As an extremely lifelike robot impersonation, dad is sitting on the couch, behaving like dad, being dad. Making a happy family home. As if he never left. The Ultimate Android.

\section{After thoughts: An industry in the making}

Ab's profession was to construct and administer vocational interest tests on Makers Island. Open- minded as he was, he offered the people there new job perspectives. Let's have a look at what his 15 year old daughter Abby found when she went through some of his documents:

Hi Abby!
According to your ranking of the statements on my test, you have an interest in graphic, interaction, multimedia, and game design; speech interfacing and language technology; semantic Web tooling, information retrieval, cluster computing and data mining; domotics, medical technology, Mechatronics, technical computer science, $\mathrm{AI}$ and software engineering; robot sensing, 3D motion-tracking and computational vision; product-service design, marketing, and business modeling. You could become a technical robot programmer, interaction designer, conversation analyst, or a robot liability lawyer. The market needs robot engineers in the medical domain, education, hospitality, etc. And not before long, HRM will be short for HumanRobot relationships Manager. My guess - that suits you fine!

What will software engineers of the future do? They will focus on embedded software (for the IoT) and on communication protocols. System and network designers and robot system-managers will work on health applications in home automation and will do field measurements that are spooled real- time to the Ultimate Android. Big Data analysts write algorithms for image-based databases and machine recognition (e.g., Deep Learning) tells the Ultimate Android what a bottle of milk is and what a tomato. For chats about the weather, robot conversation writers link up with IBM's Deep Thunder, ${ }^{19}$ which uses multiple data sources to do hyper-local weather forecasts. Nice for grandma and for the urban farmer, working on the roof of her flat. Electrical engineers will work on energy depletion. Lead-acid storage batteries are notoriously weak but robots are dependent on them. How can robots be optimized for their energy consumption? What are alternatives for conventional batteries? People like to feed their social robots (really!): A button-cell biscuit for robots, perhaps?

People in industrial automation work on the optimization of mass production of customizable PAL parts, its logistics systems, its regulation and control technology. Industrial robots assemble their social counterparts. Social robots must be integrated in many different organizations, each with their own time schedules and IT infrastructure: schools, care farms, entertainment parks, hotels, etc. We need people for that too. Well Abby, here is a list of professions that do not exist yet but you could be the person that brings them to life:

Anthrobologist- Studies the role of humans and robots in diverse cultures (including occupational) to inform the culture-specific design of (aspects of) robots

Artificial Sales Representative - Does telemarketing through robots. Can also act as a Marketing Robo Brand Manager

Autonomous Android Legislator - Juridical person (lawyer or judge) specialized in the writing of legislation (i.e. privacy, liability, intellectual property, and insurance), responsible for the execution of these laws, or the defense of people and autonomous machines that fall under those laws. An example is a Family-with-robot Practice Lawyer

Biomedical Cyborg Engineer - Develops bionic parts for impaired humans and provides machines with neuro-chips to connect silicon circuits to living brain cells

Data-Vault Security Specialist Makes sure personal data is safe from hacks by government or large corporates. Specialized in counter spyware. Controls systems for unwillingly farming out computational 
activity to 3rd parties. Advices on authorization protocols HumanRobot Interaction Counselor - Treats pathological cases of robophobia and/or robophilia. Also specializes in human-robot labor relations and robot-related occupational therapy

Electro-mechanic Repair Technician - Service professional who patches-up robots on the fly and does regular maintenance work; a Medical Robot Technician is an instantiation of this Government Security or Intelligence Agent - Adversary of the Data-Vault Security Specialist

Personal Cloud Librarian - Curates all the information individuals leave after they deceased and conserves and keeps accessible legacy systems and media that carry such personal information

Plastic Similarity Surgeon - Makes the user look more like his/ her robot

Robot App Maker - Creates software applications to install on a robot platform such as a Financial Planner Application, Administrative Assistant Application, a Weather Forecast App, a Nurse App, Food Distributor App, Personal Trainers App, Massage Therapy (Figure 2)

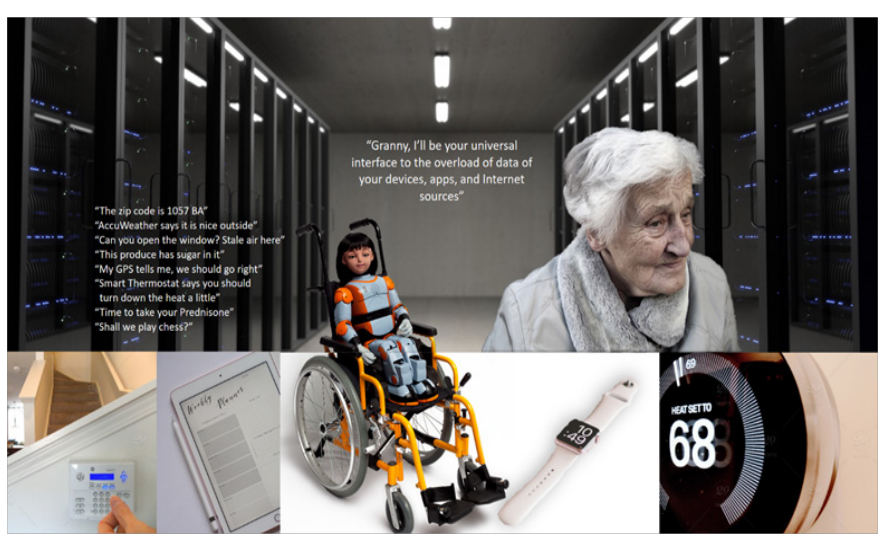

Figure 2 Abby at age 90 , supported by Alice with a wheelchair as walker rollator. ${ }^{20}$

Robot Art and Antique Dealer - Sells art created by robots, such as fractal paintings or milled stone sculptures. Also sells outmoded robot models. Related to a tech-curator in art, history, or science museums

Robot Dance Animator - Designs and implements the 'choreography' of robot movements; in stage performance as well as household chores. Usually has a background in physical therapy and dance. Works with exoskeletons as well. Often collaborates with a Robot Fashion Designer when machines enter the catwalk

Robot Science Teacher (H.S., M.S.) - Teaches basic and advanced courses in schools to bring all levels of society up to speed with (social) robotics

Simulated-Script Writer - Writes texts for conversational AI, writes screen plays for robot soap-series that feature Television and Theater Actroids. Also reports on robot sports (e.g., robot-soccer championships, robot combat competitions). Often involved in reality soaps shot through an Actroid's camera-eyes
Speech Synthesis Pathologist - Analyses speech interfaces for flaws in the language (pronunciation, pause, accent, pitch) and does proposals for adaptation to the hearing condition of the listener (i.e. elderly or impaired)

Virtual Unemployment Inspector - Virtual because not needed. Those who work have a fulfilling job, those who don't are on holiday because robots took over all of their donkeywork

Love you, Abby

Dad

\section{Acknowledgements}

My research project was partially or fully sponsored by (Ministry of Education, Culture, and Science) with grant number (NWO 646.000.003). In case of no financial assistance for the research work, provide the information regarding the sponsor.

\section{Conflict of interest}

The authors declare there is no conflict of interest.

\section{References}

1 ASIMO.

2 Geminoid F. Meet Geminoid F, a Smiling Female Android. IEEE Spectrum. 2010.

3 Hanson Robotics.

4 Boston Dynamics.

5 IBM builds a smarter planet.

6 Rob Speer. ConceptNet 5.4.2. Python.

7 http://www.teamfast.nl/nl/over-ons/

$8 \mathrm{http}: / /$ www.nerdalize.com/

9 Johan F Hoorn. The Robot Brain Server: Design of a Human-Artificial Systems Partnership. Intelligent Human Systems Integration. Springer International Publishing. 2017.

10 Isabella's First Steps" Interactive Walking Baby Doll.

11 Nabaztag.

12 Mind Modeling.

13 Johan F Hoorn, Matthijs Pontier, Ghazanfar F. Siddiqui. Coppe'é concoction: Similarity and complementarity among three affect-related agent models. Cognitive Systems Research. 2012. p. 33-49.

14 https://benjamins.com/\#catalog/books/lal.12/main.

15 Creative Confluence.

$16 \mathrm{https}: / /$ www.realdoll.com/

17 http://www.imdb.com/title/tt0470752/

$18 \mathrm{http}: / / w w w . i m d b . c o m / t i t l e / t t 1798709 /$

19 http://www-03.ibm.com/ibm/history/ibm100/us/en/icons/deepthunder/

20 Data Farm: Manuel (CC0 License) (https://static.pexels.com/ photos/325229/pexels-photo-325229.jpeg) 
Woman: Pixabay (CC0 License) (https://static.pexels.com/photos/70578/ dependent-dementia-woman-old-70578.jpeg)

Robot: Wetzer and Berends (CC0 License)

Watch: freestocks.org (CC0 License) (https://static.pexels.com/photos/213622/ pexels-photo-213622.jpeg)

Thermostat:@nikmock (Royalty-free-images) (https://res.cloudinary.com/ twenty20/private_images/t_watermark-criss-cross-10/v1483582866000/ photosp/06335d23-870b-4017-ab18-e1b5a7ee6332/stock-photo-technology- modern-hot-temperature-degrees-smart-thermostat-tech-using-technology06335d23-870b-4017-ab18-e1b5a7ee6332.jpg)

Planner: Jess Watters (CC0 License) (https://static.pexels.com/photos/768473 pexels-photo-768473.jpeg)

Home security: @gcarden76 (Royaly-free-image) (https://res.cloudinary. com/twenty20/private images/t watermark-criss-cross-10/v1466022846000/ photosp/30bd1909-2a26-42a6-8bb0-0b104b4aab99/stock-photo-safetysecurity-family-signal-home-police-secure-alarm-help-30bd1909-2a26-42a68bb0-0b104b4aab99.jpg) 